\title{
Correlation Between Pretreatment FDG-PET Biological Target Volume and Anatomical Location of Failure after Radiation Therapy for Head \& Neck Cancers
}

\author{
Daniel E. Soto, M.D., M.S. ${ }^{\star}$, Marc L. Kessler, Ph.D. ${ }^{*}$, Morand Piert, M.D.†, and Avraham \\ Eisbruch, M.D. \\ "Department of Radiation Oncology, University, of Michigan, Ann Arbor, MI \\ †Division of Nuclear Medicine, Radiology Department, University of Michigan, Ann Arbor, MI
}

\begin{abstract}
Background and Purpose: To assess whether the pretreatment FDG-PET defined biologic target volume (PET-BTV) correlates with the anatomical sites of loco-regional failure (LRF) after RT for head \& neck cancer (HNC).

Materials \& Methods: We retrospectively identified $61 \mathrm{HNC}$ patients treated definitively with either 3-D CRT or IMRT who had a pre-therapy PET/CT. The GTV and high risk CTV 1 definitions included composite data obtained from diagnostic CT, PET/CT, physical examination, and MRI when available. The median $\mathrm{CTV}_{1}$ dose was $70 \mathrm{~Gy}$. 95\% received chemotherapy. For patients with LRF, a recurrence volume ( $\mathrm{Vr}$ ) was identified and was mapped to the pretreatment planning $\mathrm{CT}$ and pretreatment PET scan.
\end{abstract}

Results: At a median follow-up of 22 months, 15\% (9/61) patients had LRF. For patients with a LRF, 100\% (9/9) of failures were inside the GTV. One of nine [11\% (95\% CI: 3\%-45\%)] had Vr which mapped outside of the pretreatment PETBTV, while 8/9 patients had Vr within the PETBTV. Predictors of LRF in our series included GTV volume ( $\mathrm{p}=0.003)$, but not mean SUV $(\mathrm{p}=0.13)$ or max SUV $(\mathrm{p}=0.25)$.

Conclusions: Following treatment in which the GTV was defined based on the composite of imaging and physica examination, the majority, but not all, LRF occurred within the PET-BTV. These results support an important, but not exclusive, role of FDG-PET in defining the GTV.

\section{Keywords}

Positron-Emission Tomography; Radiotherapy; Head and Neck Neoplasms

\section{Introduction}

Following definitive RT the majority of HNC failures are infield and occur within the $95 \%$ dose coverage region [1-5]. In order to improve treatment outcomes a better understanding of why these infield failures occur is needed. One possible explanation is that tumor cells within the gross tumor may be more resistant to RT due to biological factors such as hypoxia [6]. FDG-PET uptake may be a surrogate for hypoxia and it has been shown to

Corresponding Author: Daniel E. Soto, M.D., M.S. Department of Radiation Oncology University of Michigan 1500 E. Medical Center Drive B2 C490, Box 0010 Ann Arbor, MI 48109-0010 Phone (734) 936-4288 Fax (734) 763-7370 E-mail: dsoto@med.umich.edu.

Conflict of Interest Notification: None. No conflicts of interest exist. 
predict treatment response and loco regional failure (LRF) [7-9]. In addition, FDG-PET defined tumor volumes have been reported to be smaller than CT defined ones [10-15] and several others have suggested PET alone based methods to define the GTV. An examination of the exact site of LRF relative to pre-treatment PET defined target may shed light on the utility of PET as a vehicle to define the GTV. However, whether LRFs occur within the pretreatment PET-avid tumor site is not known. In this study, we have registered pretreatment and failure imaging scans to determine if the exact anatomical site of LRF coincides with the pretreatment PET defined tumor site.

\section{Materials \& Methods}

\section{Patients}

We obtained IRB approval from University of Michigan Cancer Center to retrospectively examine definitively treated RT HNC patients. Between 1/2001 and 6/2006, 84 patients with squamous cell $\mathrm{HNC}$ of mucosal origin treated with RT were found to have an available pretreatment in house PET/CT scan. We excluded patients with previous surgery or previous RT and focused our study on the 61 treated with definitive RT. Details of patient and tumor characteristics are provided in the Table.

\section{Therapy}

Our policies for target delineation and neck nodal coverage has been previously described [16]. The targets included gross tumor volume (GTVs) and clinical target volumes (CTVs) which consisted of areas within close proximity of the primary tumor as well as neck nodal regions at risk for sub clinical disease. GTVs were delineated using both radiologic imaging (CT, PET/CT, and MRI) as well as clinical data based on physical exam, indirect fiberoptic endoscopy, and the reports of direct endoscopy performed by surgeons. CTVs were defined utilizing previously noted guidelines [16]. GTVs and CTVs were expanded uniformly by $3-5 \mathrm{~mm}$ to generate planning target volumes (PTVs). The median prescribed doses to the PTV encompassing the GTV was 70Gy (range: 66-74.5Gy) and the dose range for PTVs encompassing sub-clinical targets 50-63Gy. Ten patients received hyperfractionated RT. Median number of fractions was 35 (range 33-59). Concurrent chemotherapy was delivered to $95 \%$ patients. 57/61 received platinum-based therapy. All patients in this series completed the treatment. Treatment factors are described in the Table.

\section{Follow-up and end points}

Base on institutional practices all patients had clinical follow-up by head and neck surgeons and radiation oncologist every 6-8 weeks for the first 2 years. Follow up then occurred every 3 months for the $3^{\text {rd }}$ year and every 6 months there after. Radiologic evaluation was performed as clinically indicated.

\section{FDG-PET/CT imaging}

FDG-PET/CT scans were obtained as part of the clinical staging procedures including intravenous contrast. Many of these studies were performed before treatment simulation, therefore were done without an immobilization mask or the treatment table adaptor. PET/CT imaging was performed on a Siemens Biography BGO-crystal tomograph with a $15.8 \mathrm{~cm}$ filed of view. After intravenous injection of 8-10 mCi or FDG, patients lay comfortably in a quiet dimly lit room. Image data were obtained from skull base to proximal thighs with arms down starting 60 minutes after tracer injection. Prior to emission imaging ( $5 \mathrm{~min}$ per bed position, $128 \times 128$ matrix), which was performed in three-dimensional mode (3D), a "lowdose" CT scan was acquired using the same axial coverage as for PET. The CT tube parameters used were $130 \mathrm{keV}$ and 80-100 mAs. 
PET image data were reconstructed iteratively based on ordered-subset expectation maximization (OSEM) with four iterations and eight subsets converting Hounsfield units (HU) to linear attenuation coefficients for $511 \mathrm{keV}$ using a single CT energy scaling method based on a bilinear transformation [17]. Transaxial and axial resolution using a post reconstruction $5 \mathrm{~mm}$ Gausian filter is 4.9 and $5.2 \mathrm{~mm}$ full-width at half-maximum (FWHM) respectively. The final pixel size of the PET images in $\mathrm{x}, \mathrm{y}, \mathrm{z}$ directions was: $0.52 \times 0.52 \times$ $0.24 \mathrm{~cm}$. The CT matrix was $512 \times 256$ pixels. The slice thickness for reconstructed PET images and CT images were both: $0.24 \mathrm{~cm}$.

\section{PET Data Analysis}

The standardized uptake value (SUV), an index of glucose metabolism, which equals the FDG uptake in each voxel normalized by the injected dose and body weight, was calculated in tumor tissues. A $1.5 \mathrm{~cm}^{2}$ circular region of interest (ROI) was centered in the area of highest tumor FDG uptake (primary tumor and/or lymph nodes) and the mean SUV and max SUV recorded.

\section{Registration of recurrence volumes, planning CT and pretreatment PET/CT}

We have previously detailed our method of characterizing failures as in-field, marginal, or out-of-field [5]. Briefly a recurrence volume (Vr) was identified on the post treatment CT, $\mathrm{PET} / \mathrm{CT}$, or MRI. Vr was then mapped to the planning CT scan to determine its isodose line (IDL) coverage. "In-field" failures were defined as having a $\mathrm{Vr} \geq 95 \%$ within the $95 \%$ IDL. "Marginal" failures had a Vr 20-95\% within the 95\% IDL. "Out-of-field" failures had a $\mathrm{Vr}<20 \%$ inside the 95\% IDL.

\section{Image Registration and Contour Transfer}

In order to transfer contours of structures drawn on the PET/CT data to the treatment planning CT the CT data from the PET/CT study were geometrically registered with the treatment planning CT using in-house software that permits both rigid and deformable registration of two image studies [18]. The PET/CT data were transferred to U-M Plan, our in-house treatment planning system using a standard DICOM mechanism. The CT data from the PET/CT were registered to the treatment planning CT using a multiresolution B-spline transformation model $[18,19]$ and a mutual information similarity metric $[20,21]$. The algorithm first registers the two datasets using a rotate-translate transformation model and uses this result as the starting point for a course to fine B-spline registration [19]. During the registration process both the resolution of the image datasets and the spacing of the B-spline grid are successively refined.

The output of the registration process is a geometric transformation which maps points from the PET/CT to the treatment planning CT. This transformation was applied to vertices of the surface generated from the contours from the PET/CT data to map the surface to the treatment planning CT. The transformed surface was intersected along the image planes of the treatment planning CT to generate contours of the structures originally drawn on PET/ CT images.

In addition to transferring the contours of the recurrence volume from the PET/CT to the treatment planning CT, the contours for the external surface and mandible were also transferred as partial validation of the computed geometric transformation.

\section{Statistical analysis}

Utilizing the Kaplan-Meier method, survival, LRF, and DF rates were calculated from the start of RT. We performed D'Agostino \& Pearson omnibus normality test and subsequently performed unpaired $t$ - tests and Mann-Whitney $\mathrm{U}$ tests. Ninety five percent confidence 
intervals where reported where indicated. Two sided p-values were utilized. Statistical analysis was performed using GraphPad Prism version 5.0, GraphPad Software, San Diego, Ca USA, www.graphpad.com.

\section{Results \\ Patient Outcomes}

The median follow-up was 22 months, range 4-72 months. At last follow-up 14 of 61 patients had died. Ten patients had died with disease recurrence. Of the remainder; one died with a secondary lung primary, one with carotid blowout and two from causes unknown. 9/61 had a LRF, of whom but four were isolated LRF. Five patients had isolated DF while 5 had a LRF and DF. The two year actuarial LRF rate and isolated DF rate were 17\% and 5\% respectively.

\section{Patterns of Failure}

The Median Vr was $15.1 \mathrm{~cm} 3$ (range 2.9-189.6). For patients with a LRF, 100\% (9/9) of failures were in-field. Of the in-field failures, one of nine, $11 \%$ (95\% CI: 3\%-45\%) had $\mathrm{Vr}$ which mapped outside of the pretreatment PET-BTV. However, it lay within the GTV which had been determined using the $\mathrm{CT}$ and physical examination in addition to the PET (Figure 1). For the remainder of the patients, $8 / 9$ had Vr within the PET-BTV (Figure 2). All failures occurred in the high dose PTV, median dose was 70Gy (range: 70-74.5Gy). In addition, they were noted to map within the CT/MRI abnormality, including the recurrence which mapped outside the PET-defined BTV.

\section{Predictors of Outcomes}

Comparison of disease free patients vs. those with any failure revealed that the mean GTV significantly differed at $122 \mathrm{~cm}^{3}$ (95\% CI: 99-144) vs. $229 \mathrm{~cm}^{3}$ (95\% CI: 152-307) p=0.003 respectively. The mean SUV for patients with and without LRF was 11.6 (95\% CI: 7.0-16.2) and 12.5 (95\% CI: 7.8-17.2) respectively ( $\mathrm{p}=0.13)$, indicating that the pretreatment tumor mean SUV did not correlate with LRF. Results did not differ when examining the max SUV $(\mathrm{p}=0.25)$ (Table).

\section{Discussion}

In this study we have demonstrated that all except one LRF occurred in the pretreatment PET-BTV. Thus, FDG-PET is important but should not be the sole instrument used in GTV definition. Some institutions have examined the feasibility of GTV delineation based only the FDG avid PET-CT volume [22,23]. Given that we observed one of nine LRF (11\%, 95\% CI: 3-45\%) outside the PETBTV, the confidence intervals suggest that if the GTVs are based solely on FDGPET, under treatment could occur in up to $45 \%$ of patients. Madani and colleagues have recently reported similar findings with 33\% (3/9) LRF outside the PETBTV [24]. Both our series and Madani's likely underestimate the true risk of failures outside the PET-BTV as all patients in these series had GTVs defined beyond the PET-BTV.

Gross pathology studies have demonstrated that no single imaging modality can depict all gross tumor extension. However, when used together MRI, CT, and PET each can add complimentary data to improve tumor delineation [25]. While most studies examining PET alone based GTVs are usually smaller then their counterpart CT based GTVs, in 25\% of patients of cases the additional information obtained from PET can increase the GTV compared to CT alone based GTVs [10-13,15,26-28]. In addition, this number can increase to up to $64 \%$ depending on the SUV threshold used [28]. These results emphasize the value of using PET as an additional source of information to assure adequate target coverage. 
However, despite this improvement in target delineation, imaging data alone still misses up to $13 \%$ of the gross tumor volume [25], with the sub-mucosal extent tumor underestimated by all imaging modalities. This emphasizes the need to use physical examination as an additional source of data for GTV delineation.

PET defined BTVs may be an area that warrants augmented therapy, such as RT boost dose escalation $[6,24,29]$. At this time we do not know if higher RT doses to these targets could improve failure rates and outcomes. However, our data supports the need to explore this as the majority of failures mapped to the pretreatment PET abnormality. Others have previously shown that dose escalation of the PET abnormality is feasible in select patients [24,30,31]. Such areas could be part of a simultaneous integrated boost [32,33]. It is unclear whether these targets for dose escalation should be defined by, visual interpretation [11], a threshold intensity plateau [28], or if the boost dose intensity should be specifically tailored to each imaging voxel intensity (i.e. painting by numbers) [34]. At this time the optimal methods for PET volume segmentation [28] and reconstruction algorithms [35] have not been established, however gradient based methods appear more promising when examining gross tumor specimens [36]. In addition, it is not clear if boost should be given to the pretherapy BTV or to the residual uptake after several weeks of therapy [37].

There are several limitations in our retrospective study that warrant discussion. First, only a limited number of treatment failures were observed in this study. Thus our confidence interval for LRF outside the PET-BTV is quite wide ranging from 3 to $45 \%$. Another concern is that failures were heterogeneous in regards to tumor location and stage. A third limitation is that PET imaging was not performed in the treatment position, thus registration quality could have impacted our results. However, we did use deformable image registration to account for this. While our study was retrospective, we did limit the study population to patients who only had PET scans at our hospital performed on the same imaging device and a standardized protocol. This is critical as difference in scanning protocols, injection times and PET/CT machines could cause differences in maximal measured SUV [38]. Given the small number of subjects included, our results should be considered preliminary and requires verification in a larger prospective series.

Future efforts should be focused on verifying our results. In addition, other areas that warrant attention include the assessment of the FDG signal during treatment to see if they correlate with treatment outcomes [39]. Finally, different forms of biological imaging agents or protocols that examine perfusion and hypoxia $[33,40]$, may also warrant further study.

In conclusion, following treatment in which the GTV was defined based on the composite of CT, PET/CT, MRI, and physical examination findings, the majority (but not all) LRF occurred within the PET-BTV. Thus, FDG-PET should not be the sole instrument used in GTV definition. High-risk GTV volumes should be continued to be defined based on composite diagnostic CT, PET/CT, MRI, and physical examination findings.

\section{Acknowledgments}

We are indebted to Steven Kronenberg for his assistance in the generation of figures.

Acknowledgment of grant or other financial support: None

\section{References}

1. Yao M, Nguyen T, Buatti JM, et al. Changing failure patterns in oropharyngeal squamous cell carcinoma treated with intensity modulated radiotherapy and implications for future research. American journal of clinical oncology 2006;29:606-612. [PubMed: 17148999] 
2. Chao KS, Ozyigit G, Tran BN, Cengiz M, Dempsey JF, Low D. Patterns of failure in patients receiving definitive and postoperative IMRT for head-and-neck cancer. International journal of radiation oncology, biology, physics 2003;55:312-321.

3. Lauve A, Morris M, Schmidt-Ullrich R, et al. Simultaneous integrated boost intensity-modulated radiotherapy for locally advanced head-and-neck squamous cell carcinomas: II--clinical results. International journal of radiation oncology, biology, physics 2004;60:374-387.

4. Feng M, Jabbari S, Lin A, et al. Predictive factors of local-regional recurrences following parotid sparing intensity modulated or 3D conformal radiotherapy for head and neck cancer. Radiother Oncol 2005;77:32-38. [PubMed: 16154219]

5. Dawson LA, Anzai Y, Marsh L, et al. Patterns of local-regional recurrence following parotidsparing conformal and segmental intensity-modulated radiotherapy for head and neck cancer. Int J Radiat Oncol Biol Phys 2000;46:1117-1126. [PubMed: 10725621]

6. Grosu A, Piert M, Molls M. Experience of PET for target localisation in radiation oncology. British Journal of Radiology 2005;(Supplement 28):18-32.

7. Allal AS, Slosman DO, Kebdani T, Allaoua M, Lehmann W, Dulguerov P. Prediction of outcome in head-and-neck cancer patients using the standardized uptake value of 2-[18F]fluoro-2-deoxy-Dglucose. Int J Radiat Oncol Biol Phys 2004;59:1295-1300. [PubMed: 15275712]

8. Brun E, Kjellen E, Tennvall J, et al. FDG PET studies during treatment: prediction of therapy outcome in head and neck squamous cell carcinoma. Head \& neck 2002;24:127-135. [PubMed: 11891942]

9. Kitagawa Y, Sano K, Nishizawa S, et al. FDG-PET for prediction of tumour aggressiveness and response to intra-arterial chemotherapy and radiotherapy in head and neck cancer. Eur J Nucl Med Mol Imaging 2003;30:63-71. [PubMed: 12483411]

10. Paulino AC, Koshy M, Howell R, Schuster D, Davis L. Comparison of CT- and FDG-PET-defined gross tumor volume in intensity-modulated radiotherapy for head-and-neck cancer. Int J Radiat Oncol Biol Phys 2005;61:1385-1392. [PubMed: 15817341]

11. Ciernik IF, Dizendorf E, Baumert BG, et al. Radiation treatment planning with an integrated positron emission and computer tomography (PET/CT): a feasibility study. Int J Radiat Oncol Biol Phys 2003;57:853-863. [PubMed: 14529793]

12. Nishioka T, Shiga T, Shirato H, et al. Image fusion between 18 FDG-PET and MRI/CT for radiotherapy planning of oropharyngeal and nasopharyngeal carcinomas. Int J Radiat Oncol Biol Phys 2002;53:1051-1057. [PubMed: 12095574]

13. Wang D, Schultz CJ, Jursinic PA, et al. Initial experience of FDG-PET/CT guided IMRT of headand-neck carcinoma. Int J Radiat Oncol Biol Phys 2006;65:143-151. [PubMed: 16618577]

14. Andrade RS, Heron DE, Degirmenci B, et al. Posttreatment assessment of response using FDGPET/CT for patients treated with definitive radiation therapy for head and neck cancers. Int $\mathrm{J}$ Radiat Oncol Biol Phys 2006;65:1315-1322. [PubMed: 16750327]

15. Ng SH, Yen TC, Chang JT, et al. Prospective study of [18F]fluorodeoxyglucose positron emission tomography and computed tomography and magnetic resonance imaging in oral cavity squamous cell carcinoma with palpably negative neck. J Clin Oncol 2006;24:4371-4376. [PubMed: 16983105]

16. Eisbruch A, Foote RL, O'Sullivan B, Beitler JJ, Vikram B. Intensity-modulated radiation therapy for head and neck cancer: emphasis on the selection and delineation of the targets. Semin Radiat Oncol 2002;12:238-249. [PubMed: 12118389]

17. Kinahan PE, Townsend DW, Beyer T, Sashin D. Attenuation correction for a combined 3D PET/ CT scanner. Med Phys 1998;25:2046-2053. [PubMed: 9800714]

18. Kessler ML. Image registration and data fusion in radiation therapy. The British journal of radiology 2006;79(Spec No 1):S99-108. [PubMed: 16980689]

19. Kybic J, Unser M. Fast Parametic Elastic Image Registration. IEEE transactions on medical imaging 2003;12:1427-1441.

20. Viola P. Alignment by Maximization of Mutual Information. Int J Comp Vis 1997;24:137-154.

21. Maes F, Collignon A, Vandermeulen D, Marchal G, Suetens P. Multimodality image registration by maximization of mutual information. IEEE transactions on medical imaging 1997;16:187-198. [PubMed: 9101328] 
22. Schwartz DL, Ford EC, Rajendran J, et al. FDG-PET/CT-guided intensity modulated head and neck radiotherapy: a pilot investigation. Head \& neck 2005;27:478-487. [PubMed: 15772953]

23. Ford EC, Kinahan PE, Hanlon L, et al. Tumor delineation using PET in head and neck cancers: threshold contouring and lesion volumes. Medical physics 2006;33:4280-4288. [PubMed: 17153406]

24. Madani I, Duthoy W, Derie C, et al. Positron emission tomography-guided, focal-dose escalation using intensity-modulated radiotherapy for head and neck cancer. International journal of radiation oncology, biology, physics 2007;68:126-135.

25. Daisne JF, Duprez T, Weynand B, et al. Tumor volume in pharyngolaryngeal squamous cell carcinoma: comparison at CT, MR imaging, and FDG PET and validation with surgical specimen. Radiology 2004;233:93-100. [PubMed: 15317953]

26. Heron DE, Andrade RS, Flickinger J, et al. Hybrid PET-CT simulation for radiation treatment planning in head-and-neck cancers: a brief technical report. International journal of radiation oncology, biology, physics 2004;60:1419-1424.

27. El-Bassiouni M, Ciernik IF, Davis JB, et al. [18FDG] PET-CT-based intensity-modulated radiotherapy treatment planning of head and neck cancer. International journal of radiation oncology, biology, physics 2007;69:286-293.

28. Schinagl DA, Vogel WV, Hoffmann AL, van Dalen JA, Oyen WJ, Kaanders JH. Comparison of five segmentation tools for 18F-fluoro-deoxy-glucose-positron emission tomography-based target volume definition in head and neck cancer. Int J Radiat Oncol Biol Phys 2007;69:1282-1289. [PubMed: 17967318]

29. Ling CC, Humm J, Larson S, et al. Towards multidimensional radiotherapy (MD-CRT): biological imaging and biological conformality. Int J Radiat Oncol Biol Phys 2000;47:551-560. [PubMed: 10837935]

30. Chao KS, Bosch WR, Mutic S, et al. A novel approach to overcome hypoxic tumor resistance: CuATSM-guided intensity-modulated radiation therapy. Int J Radiat Oncol Biol Phys 2001;49:11711182. [PubMed: 11240261]

31. Thorwarth D, Eschmann SM, Paulsen F, Alber M. Hypoxia dose painting by numbers: a planning study. International journal of radiation oncology, biology, physics 2007;68:291-300.

32. Butler EB, Teh BS, Grant WH 3rd, et al. Smart (simultaneous modulated accelerated radiation therapy) boost: a new accelerated fractionation schedule for the treatment of head and neck cancer with intensity modulated radiotherapy. Int J Radiat Oncol Biol Phys 1999;45:21-32. [PubMed: 10477002]

33. Grosu AL, Souvatzoglou M, Roper B, et al. Hypoxia Imaging With FAZA-PET and Theoretical Considerations With Regard to Dose Painting for Individualization of Radiotherapy in Patients With Head and Neck Cancer. Int J Radiat Oncol Biol Phys 2007;69:541-551. [PubMed: 17869667]

34. Vanderstraeten B, Duthoy W, De Gersem W, De Neve W, Thierens H. [18F]fluoro-deoxy-glucose positron emission tomography ([18F]FDG-PET) voxel intensity-based intensity-modulated radiation therapy (IMRT) for head and neck cancer. Radiother Oncol 2006;79:249-258. [PubMed: 16564588]

35. Daisne JF, Sibomana M, Bol A, Doumont T, Lonneux M, Gregoire V. Tri-dimensional automatic segmentation of PET volumes based on measured source-to-background ratios: influence of reconstruction algorithms. Radiother Oncol 2003;69:247-250. [PubMed: 14644483]

36. Geets X, Lee JA, Bol A, Lonneux M, Gregoire V. A gradient-based method for segmenting FDGPET images: methodology and validation. Eur J Nucl Med Mol Imaging 2007;34:1427-1438. [PubMed: 17431616]

37. Geets X, Tomsej M, Lee JA, et al. Adaptive biological image-guided IMRT with anatomic and functional imaging in pharyngo-laryngeal tumors: impact on target volume delineation and dose distribution using helical tomotherapy. Radiother Oncol 2007;85:105-115. [PubMed: 17562346]

38. Hoekstra CJ, Paglianiti I, Hoekstra OS, et al. Monitoring response to therapy in cancer using [18F]-2-fluoro-2-deoxy-D-glucose and positron emission tomography: an overview of different analytical methods. Eur J Nucl Med 2000;27:731-743. [PubMed: 10901462] 
39. van Baardwijk A, Bosmans G, Dekker A, et al. Time trends in the maximal uptake of FDG on PET scan during thoracic radiotherapy. A prospective study in locally advanced non-small cell lung cancer (NSCLC) patients. Radiother Oncol 2007;82:145-152. [PubMed: 17258339]

40. Lehtio K, Eskola O, Viljanen T, et al. Imaging perfusion and hypoxia with PET to predict radiotherapy response in head-and-neck cancer. Int J Radiat Oncol Biol Phys 2004;59:971-982. [PubMed: 15234030] 

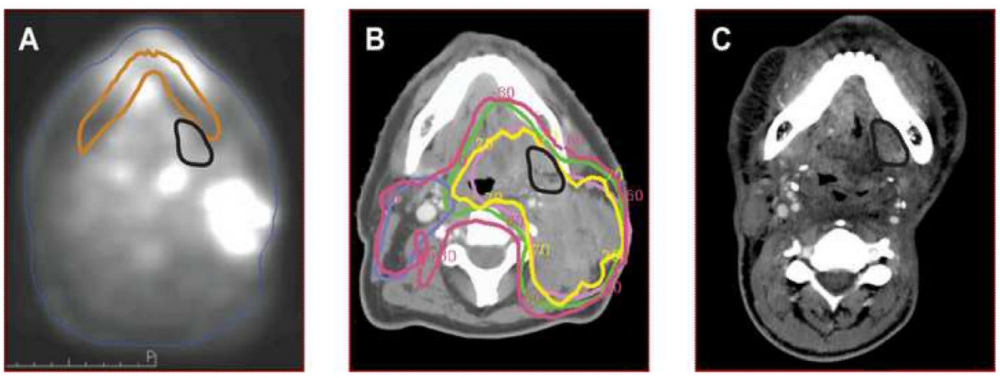

Figure 1. In-field recurrence (Vr) outside of PET-BTV

a) Pretreatment PETCT (CT data set not shown). Vr mapped in black from registered failure scan. Failure scan was rigidly registered after using mandibles. Mandible from failure scan is outlined in brown. b) IMRT treatment planning CT scan. Targets: PTV 70Gy is pink, PTV 64Gy is green, \& PTV 60Gy is blue. 70Gy iso dose line (IDL) is in yellow \& 60Gy IDL is in fuchsia. Vr is black. C) Failure CT scan with Vr in black. 

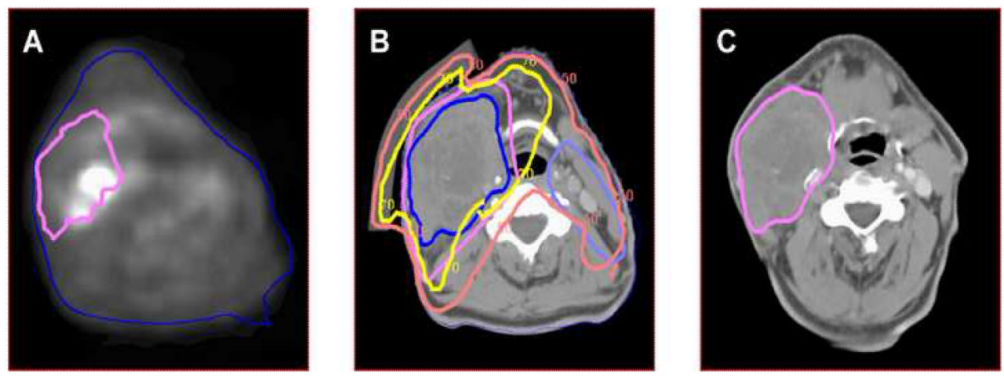

Figure 2. In-field recurrence (Vr) within PET-BTV

a) Pretreatment PET-CT (CT data set not shown). Vr mapped in pink from registered failure scan. b) IMRT treatment planning CT scan. Targets: PTV 70Gy is pink, PTV 50Gy is light blue. $68 \%$ Gy iso dose line (IDL) is in yellow \& 48Gy IDL is in fuchsia. Vr is dark blue. C) Failure CT scan with $\mathrm{Vr}$ in pink. 
Table

Patient \& Treatment Characteristics:

\begin{tabular}{|c|c|c|}
\hline & Disease Free & Failures \\
\hline No. patients & 47 & 14 \\
\hline Median Age-yrs & 56 & 61 \\
\hline Gender $\mathbf{M} / \mathbf{F}$ & $38: 9$ & $8: 6$ \\
\hline \multicolumn{3}{|l|}{ Stage } \\
\hline III & 4 & 2 \\
\hline IVA & 37 & 5 \\
\hline IVB & 6 & 7 \\
\hline \multicolumn{3}{|l|}{ Site } \\
\hline Oropharynx & 37 & 8 \\
\hline Larynx & 5 & 2 \\
\hline Unknown primary & 2 & 2 \\
\hline Hypopharynx. & 1 & 1 \\
\hline Nasopharynx & 1 & 1 \\
\hline Oral cavity & 1 & 0 \\
\hline Concurrent Chemo & $94 \%$ & $100 \%$ \\
\hline Platinum Based & $100 \%$ & $93 \%$ \\
\hline Median RT Dose-Gy & 70 & 70 \\
\hline IMRT vs 3-D & $33: 14$ & $5: 9$ \\
\hline RT Duration days & 49 & 47 \\
\hline Mean GTV $\mathrm{cm}^{3}(95 \% \mathrm{CI})$ & $121.5(99.0-144.0)$ & $229.4(152.2-306.7) *$ \\
\hline Mean SUV (95\% CI) & $11.6(7.0-16.2)$ & $12.5(7.8-17.2)^{\dagger}$ \\
\hline Max SUV (95\% CI) & $14.1(9.2-19.0)$ & $14.9(9.8-20.1){ }^{\#}$ \\
\hline
\end{tabular}

Statistically significant difference $\mathrm{p}=0.003$.

${ }^{\dagger}$ Not statistically different $\mathrm{p}=0.13$.

${ }^{\#}$ Not statistically different $\mathrm{p}=0.25$. 